\title{
NEUROPATÍA ÓPTICA ISQUÉMICA ANTERIOR NO ARTERÍTICA INCIPIENTE O PRECLÍNICA
}

\section{INCIPIENT OR PRESYMPTOMATIC NONARTERITIC ANTERIOR ISCHEMIC OPTIC NEUROPATHY}

\author{
REBOLLEDA G ${ }^{1}$, MUÑOZ-NEGRETE FJ ${ }^{1}$
}

\begin{abstract}
RESUMEN
Caso clínico: Se presenta un paciente de 62 años con neuropatía óptica isquémica anterior no arterítica (NOIANA) clásica en ojo izquierdo, y forma incipiente en ojo derecho con progresión a forma clásica a las 3 semanas. Se excluyeron otras causas de tipo ocular, orbitario, neurológico o hematológico que pudieran relacionarse con el edema papilar.

Discusión: Hayreh ha descrito recientemente la entidad NOIANA incipiente. Los criterios diagnósticos básicos son la presencia de un edema de disco sin pérdida de visión asociada en el ojo adelfo de pacientes con NOIANA. Representa el estadio clínico asintomático más precoz en la evolución de la NOIANA. No hay tratamiento para esta entidad, y para reducir el riesgo de progresión de forma incipiente a clásica se aconseja tratar los factores de riesgo de la forma clásica.
\end{abstract}

Palabras clave: Neuropatía óptica isquémica, edema de papila, NOIA, neuropatía óptica isquémica incipiente, nervio óptico.

\begin{abstract}
Case report: We report the case of a 62-year-old man with classic nonarteritic anterior ischemic optic neuropathy (NAION) in the left eye and incipient NAION in the right eye. Incipient NAION progressed to classic NAION after 3 weeks. There was no ocular, orbital, neurologic, or systemic disorder that was responsible for optic disc edema.

Discussion: Recently, Hayreh has described the new entity, incipient NAION. Diagnostic criteria include asymptomatic optic disc edema and no visual loss in the contralateral eye of patients affected by NAION. Incipient NAION represents the earliest asymptomatic clinical stage in the evolution of the NAION disease process. There is no treatment for this entity. Steps should be taken to treat risk factors for NAION to reduce the risk of progression of incipient to classic NAION (Arch Soc Esp Oftalmol 2009; 84: 151-154).
\end{abstract}

Key words: Anterior ischemic optic neuropathy, incipient anterior ischemic optic neuropathy, optic disc swelling, NAION, optic nerve.

\section{CASO CLÍNICO}

Paciente de 62 años que acudió a Urgencias por pérdida de visión en ojo izquierdo (OS), sin dolor ocular, cefalea, ni otros síntomas neurológicos acompañantes. La agudeza visual (AV) era 1 en ojo derecho (OD) y 0,1 en OS, con un defecto pupilar aferente relativo izquierdo.

Recibido: 13/10/07. Aceptado: 24/3/09.

Departamento de Oftalmología. Unidad de Glaucoma y Neuro-Oftalmología. Hospital Ramón y Cajal. Universidad de Alcalá. Madrid. España.

${ }^{1}$ Doctor en Medicina.

Correspondencia:

Gema Rebolleda

Servicio de Oftalmología. Hospital Ramón y Cajal

Ctra. de Colmenar Viejo, km 9,1

28034 Madrid

España

E-mail: grebolleda@ telefonica.net 
El examen funduscópico revelaba un edema sectorial superior en OD y un edema papilar difuso con hemorragias en llama peripapilares en OS (fig. 1a).

El examen campimétrico fue normal en OD (fig. 2), objetivándose un defecto severo difuso en OS.

La presión arterial, velocidad de sedimentación globular y analítica sanguínea fueron normales, excepto un aumento del fibrinogeno sérico, por lo que se comenzó tratamiento con acido acetilsalicílico. El estudio hematológico y neurológico realizado fue completamente normal.

A las 3 semanas el paciente acudió de nuevo a Urgencias por perdida de visión brusca en el OD, con reducción de AV a 0,3. En el examen funduscópico el edema del OD era más pronunciado y difuso, con presencia de hemorragias en llama peripapilares (fig. 1b). En la perimetría se detectó un defecto arqueado en el hemicampo inferior (fig. 2).

A los 2 meses del comienzo del cuadro, el edema de papila se había resuelto en ambos ojos (OU) (fig. 1c), siendo la AV de 0,6 en OD y 0,2 en OS. La tomografía de coherencia óptica puso de manifiesto un adelgazamiento significativo de la capa de fibras nerviosas en $270^{\circ}$ en OU.

\section{DISCUSIÓN}

Los pacientes con una neuropatía óptica isquémica anterior no arterítica (NOIANA) presentan típi- camente una pérdida de visión brusca, indolora con edema de papila, defecto pupilar aferente y alteraciones campimétricas ipsilaterales $(1,2)$.

En 1981, Hayreh describió por primera vez una fase de edema de disco asintomático, que precede al cuadro clínico clásico de NOIANA (3).

Recientemente, en 2007, Hayreh y Zimmerman revisan 60 ojos de 54 pacientes evaluados en un período de tiempo de 27 años, con el diagnóstico de NOIANA incipiente. Los criterios diagnósticos de esta entidad son los siguientes:

1. Edema disco sectorial o difuso que predomina más en un sector que otros.

2. Ausencia de pérdida de visión ni subjetiva ni objetiva asociada.

3. Ausencia de otras causas de origen ocular, hematológico, orbitario o neurológico asociadas (4).

Nuestro paciente presentó durante la fase aguda de una NOIANA clásica izquierda, un edema de disco sectorial superior en el ojo adelfo, sin pérdida de visión, con perimetría normal y sin otras causas que pudieran justificar dichos hallazgos (fig. 1); ajustándose por lo tanto a los 3 criterios diagnósticos mencionados.

Hayreh y Zimmerman no encontraron diferencias en la visión al final del seguimiento entre aquellos casos que progresaron y los que no, con una $\mathrm{AV}$ igual o mejor de 20/25 en aproximadamente el $60 \%$ de los casos. En nuestro paciente, la AV final del

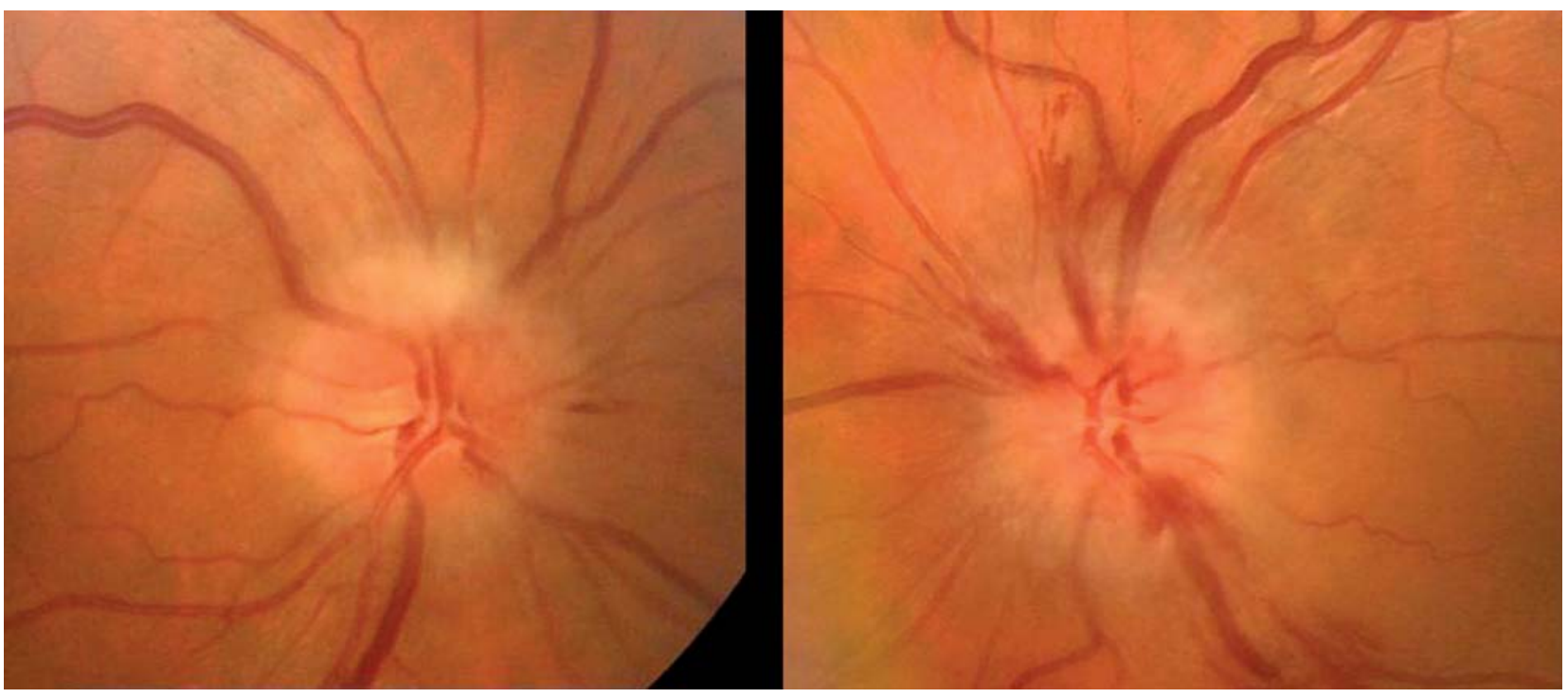

Fig. Ia: Izda: Fondo OD: Edema sectorial superior. Dcha: Fondo OS: Edema papilar difuso, hemorragias en llama peripapilares. 


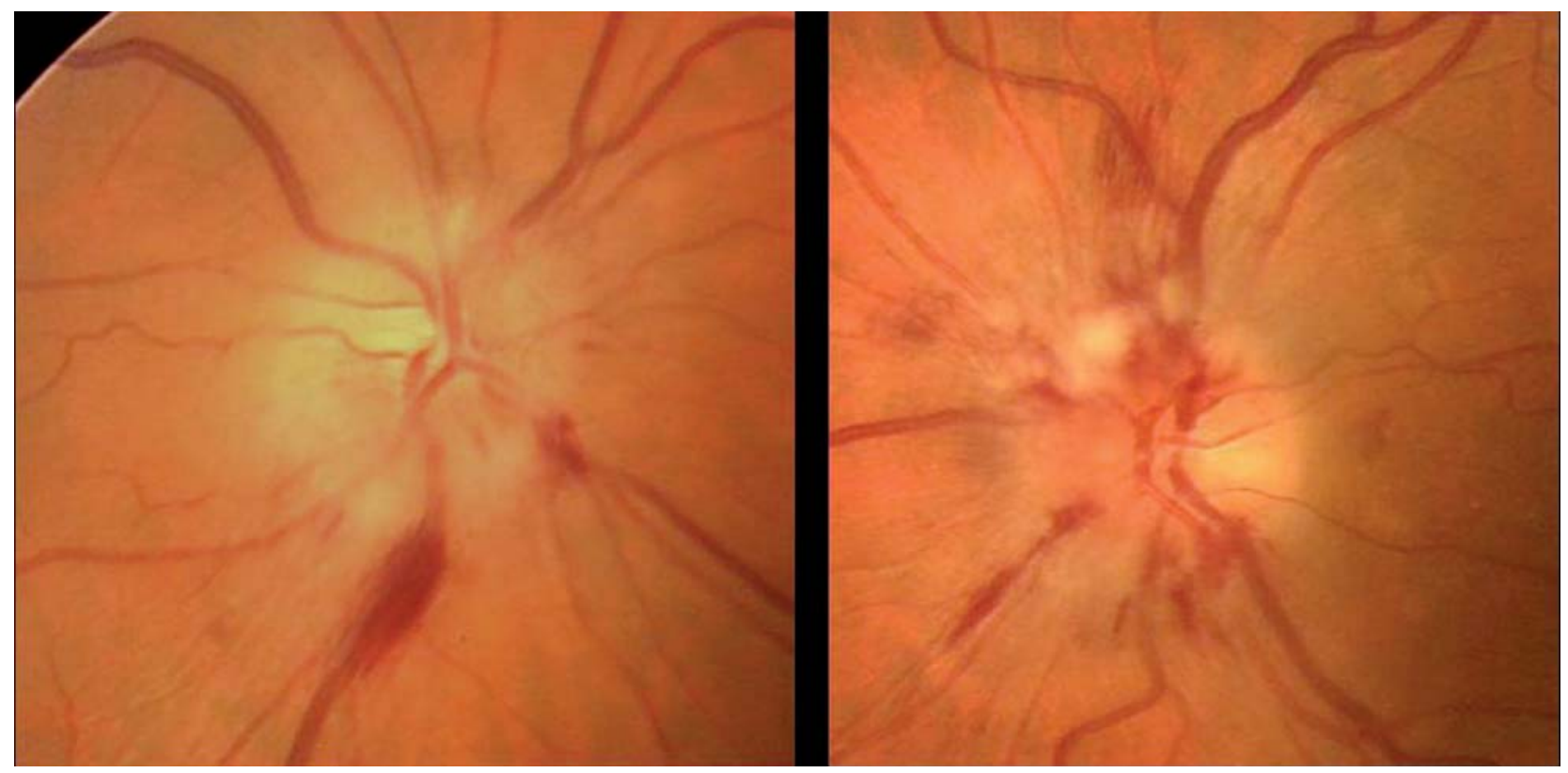

Fig. 1b: 3 semanas después de la figura la. Izda: Fondo OD: Edema difuso y nuevas hemorragias superficiales en sector inferior. Dcha: Fondo OS: El edema es más pronunciado, con presencia de más hemorragias y exudados algodonosos.

ojo derecho fue 0,6 , mejor que la del OS con la forma clásica (AV: 0,2).

Dado que el principal rasgo clínico de la forma incipiente es un edema de disco asintomático, es preciso excluir varias entidades diagnósticas. Así, habría que considerar cuadros que cursan con edema monocular como la uveítis posterior, oclusión de vena central de la retina, hipotonía ocular, uveítis posterior, neuritis óptica, vasculitis, neuropatía óptica de Leber, lesión compresiva orbitaria, e infiltración de nervio óptico; y también considerar cuadros que cursan con edema bilateral, como el aumento de presión intracraneal y la hipertensión arterial maligna. Sin embargo, la mayoría de los casos referidos cursan con pérdida de visión y/o otros signos clínicos asociados, que facilitan el diagnóstico.

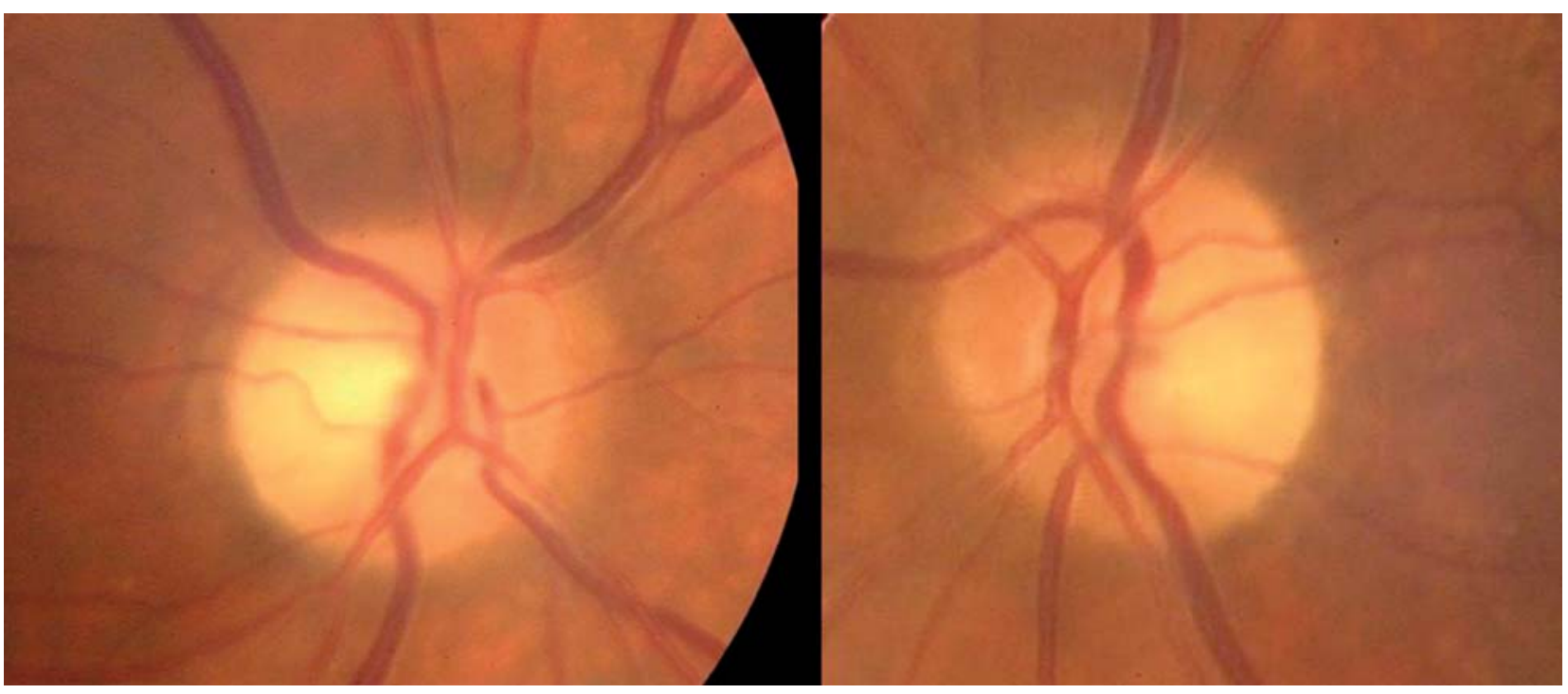

Fig. 1c: Resolución del edema en ambos ojos, a los 2 meses del episodio agudo del OS. 


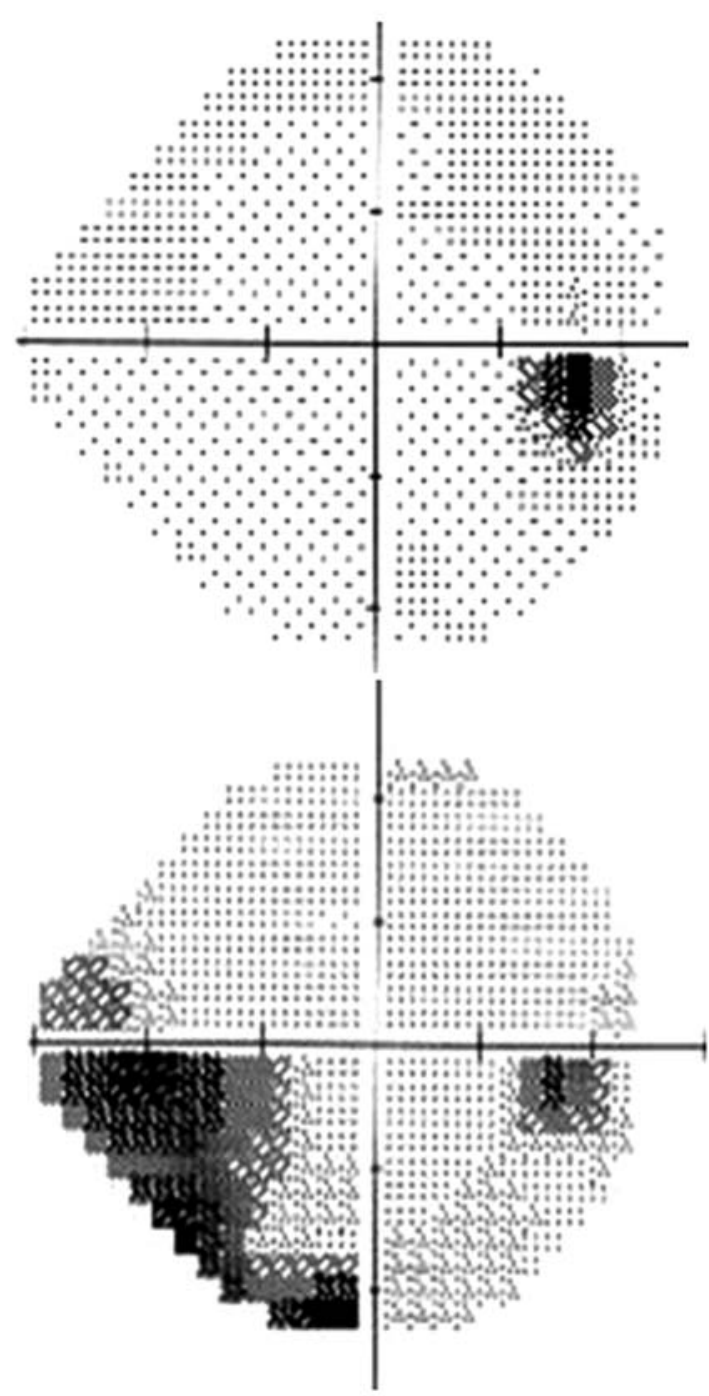

Fig. 2: Superior: Campo visual (CV) normal en $O D$ durante el episodio de neuropatía óptica isquémica aguda de OI (corresponde a imagen de fondo de la figura 1a). Inferior: CV OD 3 semanas después, mostrando un defecto arciforme inferior (corresponde a la imagen de fondo de la figura $1 b$ ).

En la serie publicada por Hayreh (4), el 55\% de los casos tuvieron una NOIANA clásica asociada en el ojo adelfo, bien simultáneamente o separada en el tiempo. El 25\% de los casos progresaban a la forma clásica en un tiempo medio de 6 semanas y el $20 \%$ lo hacían en un intervalo variable tras la resolución del episodio de NOIANA incipiente. Aquellos que progresaban eran significativamente más jóvenes que los que no lo hacían.

Nuestro paciente presentó un episodio de NOIANA clásica simultáneamente en el OS, con progresión de la forma incipiente a la clásica en 3 semanas; datos que también coinciden con los hallazgos publicados por Hayreh.

Respecto al tratamiento de esta entidad, aunque en un primer estudio piloto parecía que los esteroides podrían acelerar la recuperación (2), en la revisión posterior no se ha podido corroborar dicho efecto. En dicho estudio, Hayreh y Zimmerman (4), comparan pacientes con forma incipiente que eligen ser tratados con esteroides orales ( $80 \mathrm{mg}$ vía oral el primer día, en pauta descendente después de 2 semanas), con no tratados, sin encontrar diferencias significativas. Nuestro paciente solo recibió tratamiento con Adiro ${ }^{\circledR}$ por la hiperfibrinogenemia concomitante.

Medidas profilácticas destinadas a evitar la progresión a forma clásica, incluyen el cese de aquellas medicaciones que pueden provocar episodios de hipotensión nocturna, así como reducir la tensión ocular si está elevada o en valores límites (5).

Hayreh sostiene que todos los pacientes con NOIANA comienzan con un edema de disco asintomático, pero que sólo en raras ocasiones el oftalmólogo tiene la oportunidad de evaluarlos durante dicha fase. La presencia de una NOIANA clásica en el ojo adelfo, la progresión a forma clásica o el desarrollo de forma clásica en el seguimiento apoya el diagnóstico de esta entidad clínica.

\section{BIBLIOGRAFÍA}

1. Arnold AC. Ischemic optic neuropathy. In: Walsh and Hoyt's Clinical Neuro-ophthalmology. 6th Ed. Philadelphia: Lippincott Williams \& Wilkins; 2005; 349-384.

2. Contreras I, Rebolleda G, Noval S, Muñoz-Negrete FJ. Optic disc evaluation by optical coherence tomography in nonarteritic anterior ischemic optic neuropathy. Invest Ophthalmol Vis Sci 2007; 48: 4087-4092.

3. Hayreh SS. Anterior ischemic optic neuropathy. Optic disc edema an early sign. Arch Ophthalmol 1981; 99: 10301040.

4. Hayreh SS, Zimmerman B. Incipient nonarteritic anterior ischemic optic neuropathy. Ophthalmology 2007; 114: 1763-1772.

5. Hayreh SS, Podhajski PA, Zimmerman B. Role of nocturnal arterial hypotension in optic nerve head ischemic disorders. Ophthalmologica 1999; 213: 76-79. 